\title{
Islamische Theologie, islamischer Religionsunterricht - Kritische Anmerkungen zur Funktion und Praxis der neu gegründeten Beiräte
}

\author{
Michael Kiefer
}

\section{Einleitung}

Während in den Feuilletons nach wie vor mit Verve darüber gestritten wird, ob der Islam zu Deutschland gehört, hat die Schul- und Bildungspolitik in den vergangenen zwei Jahren zu dieser Frage eindeutige Fakten geschaffen, die keinen Zweifel daran aufkommen lassen, dass der Islam in Hochschullehre und schulischer Ausbildung einen festen Platz einnehmen wird.

Jüngstes Beispiel ist die Einführung eines islamischen Religionsunterrichts in Nordrhein-Westfalen im Schuljahr 2012/2013, der sukzessiv die bei muslimischen Verbänden wenig geschätzte Islamkunde ablösen soll. ${ }^{1}$ Folgt man der Sichtweise des nordrhein-westfälischen Bildungsministeriums, stellt das neue Fach eine echte Innovation dar, da die muslimischen Organisationen des Landes bei der inhaltlichen Gestaltung des Faches und der Zulassung der Lehrkräfte erhebliche Mitspracherechte geltend machen können. Ähnlich will ab dem Schuljahr 2013/2014 auch Niedersachsen verfahren. Auch hier hat man in Übereinstimmung mit den muslimischen Verbänden eine Beiratslösung etabliert. Die Einführung eines Religionsunterrichts im Sinne des Artikels 7 Abs. 3 GG, der vorsieht, dass der Religionsunterricht in Übereinstimmung mit den Grundsätzen einer Religionsgemeinschaft zu erteilen ist, gilt damit als gesichert. Nicht unerwähnt bleiben sollten im schulischen Kontext die hessischen Bemühungen. Nach einem langwierigen Gutachterverfahren plant die Landesregierung

\footnotetext{
${ }^{1}$ Vgl. den Beitrag zum Islamischen Religionsunterricht von Klaus Spenlen (S. 307-334) in diesem Sammelband.
} 
für das kommende Schuljahr gleichfalls die Einführung eines islamischen Religionsunterrichts, der allerdings unter Auslassung einer Beiratslösung in direkter Kooperation mit Islamverbänden realisiert werden soll.

Bei der institutionellen Integration des Islam sind an einigen deutschen Universitäten gleichfalls große Anstrengungen zu verzeichnen. Als Initialzündung wirkten im Jahr 2010 die Empfehlungen des Wissenschaftsrates (WR), der an mehreren universitären Standorten die Einführung sogenannter islamischer Studien empfahl, die im Rahmen von neu zu gründenden Instituten an jeweils philosophischen oder kulturwissenschaftlichen Fakultäten angesiedelt werden sollen. ${ }^{2}$ Die Empfehlungen fanden rasch die Zustimmung der Bundesregierung, die Finanzmittel in Höhe von 20 Millionen Euro für die Implementierungsphase bereitstellte. Zwischenzeitlich können die Universitätsstandorte Münster, Osnabrück, Frankfurt (Gießen), Erlangen und Tübingen Institutsgründungen vorweisen.

An erster Stelle zu nennen ist hier die Universität Osnabrück, die bereits vor vier Jahren das Zentrum für interkulturelle Islamstudien (ZIIS) gründete. Im Institut sind mittlerweile vier Professuren, eine drei Forschungsstellen umfassende Postdoc-Gruppe und sechs wissenschaftliche Mitarbeiter tätig. In der letzten Ausbaustufe soll die islamische Theologie sieben Professuren umfassen. Münster, das nach dem Willen des BMBF eng mit Osnabrück kooperieren soll, gründete ebenfalls ein Institut. Das Zentrum für Islamische Theologie verfügt derzeit über eine Professur und ein Graduiertenkolleg. Zwei weitere Juniorprofessuren sollen in naher Zukunft folgen. Beachtliche Ausmaße hat mittlerweile auch das Institut für Studien der Kultur und Religion des Islam an der Goethe Universität Frankfurt am Main erreicht. Das Institut umfasst vier Professuren, eine vier Wissenschaftler umfassende Postdoc-Gruppe und vier wissenschaftlichen Mitarbeiter. Im März 2012 hat die Friedrich-Alexander Universität Erlangen das Department für Islamische Religiöse Studien geründet. Derzeit umfasst das Department eine Professur. Drei weitere Professuren sollen in Jahresfrist eingerichtet werden. Schließlich wäre noch das Zentrum für islamische Theologie der Eberhard Karls Universität Tübingen anzuführen, das am 16. Januar 2012 eröff-

\footnotetext{
${ }^{2}$ Vgl. WR 2010.
} 
net wurde. Bereits im Wintersemester 2012 soll die Lehre von drei Lehrstühlen und zwei Juniorprofessuren verantwortet werden.

Angesichts dieser beeindruckenden Fakten kommt man nicht umhin zu konstatieren, dass Bund und Länder in den vergangenen zwei Jahren außerordentlich große Anstrengungen unternommen haben, um die Akademisierung und schulische Beheimatung des Islam voranzutreiben. Dieser unstrittig positive Befund kann jedoch nicht darüber hinwegtäuschen, dass zur Etablierung einer islamischen Theologie an philosophischen oder kulturwissenschaftlichen Fakultäten und den Mitwirkungsmöglichkeiten der muslimischen Verbände in den sogenannten Beiräten durchaus kritische Fragen gestellt werden können.

Ein erstes nicht unerhebliches Problem betrifft die Verortung der neuen Wissenschaft an der Universität. Die Gründung einer „bekenntnisorientierten“ islamischen Theologie an einer philosophischen Fakultät verwischt die Grenze zur Islamwissenschaft. Die Islamwissenschaft betrachtet ihre Gegenstände frei von religiös-normativen Einschränkungen aus einem neutralen Erkenntnisinteresse, und die Lehrenden benötigen keine Lehrerlaubnis. Bei der Einstellung des lehrenden Personals zählt alleine die fachliche Kompetenz. Anders hingegen verhält es sich mit der islamischen Theologie. Diese betreibt ihre Forschung aus der Binnenperspektive der Religion und ist durchaus mit normativen Grenzen behaftet. Hinzu kommt, dass die Vertreterinnen und Vertreter der Lehre im Einstellungsprozess das Placet einer Religionsgemeinschaft benötigen. ${ }^{3}$ Der Islamwissenschaftler Rainer Brunner vertritt ausgehend von dieser Sachlage die pointierte Ansicht, dass allein aus erkenntnistheoretischen Erwägungen eine Theologie an einer Philosophischen Fakultät nichts zu suchen habe. ${ }^{4}$

\section{Beiräte als Vertreter einer islamischen Religionsgemeinschaft an der Hochschule}

Wer bekenntnisorientierte Islamische Studien an deutschen Universitäten etablieren möchte, braucht eine Religionsgemeinschaft, denn ein bekenntnisneutraler Staat kann die Verantwortung für die Inhalte eines Theologiestudiums

\footnotetext{
${ }^{3}$ Vgl. Michael Kiefer 2011, S. 37.

${ }^{4}$ Vgl. Rainer Brunner, Die Empfehlungen des Wissenschaftsrats zur Einführung des Fachs „Islamische Studien" aus der Sicht eines Islamwissenschaftlers.
} 
schwerlich übernehmen. Diese Grundposition vertreten nahezu alle Religionsverfassungsrechtler. Mit Blick auf die Mitwirkungsrechte der Kirchen ist diese Position auch für Nichtjuristen durchaus nachvollziehbar. In den christlichen Theologien ergeben sich die Partnerschaften aus historisch gewachsenen staatskirchlichen Verhältnissen. Die Kirchen nehmen ihre Mitwirkungsrechte in der Regel über einen Bischof wahr. Da bei den Muslimen etablierte kirchenähnliche Strukturen bekanntlich nicht vorkommen, bestand und besteht ein gravierendes Problem bei der Auswahl der Partner auf muslimischer Seite. Die altbekannten Fragen lauten unter anderem: Wer repräsentiert den Islam, und welche Strömungen sind zu berücksichtigen?

Diese Fragen wurden vor allem in Bezug auf den islamischen Religionsunterricht in den vergangenen zwei Dekaden außerordentlich kontrovers diskutiert. $\mathrm{Zu}$ einer wirklich einvernehmlichen Lösung mit allen Beteiligten ist es bislang in keinem Bundesland gekommen. Der Wissenschaftsrat, der sich dieses Sachverhalts sehr wohl bewusst ist, sieht als Mitwirkungsorgane der muslimischen Religionsgemeinschaften sogenannte Beiräte vor. Die Zusammensetzung der Beiräte „sollte dem Selbstverständnis der Muslime, der Vielfalt ihrer Organisationsformen in Deutschland sowie den Anforderungen an theologische Kom-


Mitwirkung des Koordinationsrats der Muslime (KRM) vor. Mit Blick auf eine denkbare Pluralisierung des Islams sollten die Beiräte sich aber auch für „neue muslimische Organisationen" offen zeigen. ${ }^{6}$

\subsection{Befugnisse der Beiräte}

Nach Auffassung des WR ergeben sich die umfangreichen Mitwirkungsrechte bzw. Befugnisse einer Religionsgemeinschaft bei der Ausgestaltung einer an Universitäten gelehrten islamischen Theologie aus dem Verfassungsrecht. Hierzu zählen:

- Mitwirkung an der Gründung eines Instituts für muslimische Studien

- Zustimmung zur Einrichtung islamisch theologischer Studiengänge

- Mitwirkung an der Ausarbeitung der neuen Studiengänge

\footnotetext{
${ }^{5}$ WR 2010, S. 80.

${ }^{6}$ Ebd.
} 
- Mitwirkung bei der Änderung oder Aufhebung von Studiengängen

- Beteiligung in den Berufungsverfahren.

Von zentraler Bedeutung ist insbesondere die Beteiligung der Beiräte in den Berufungsverfahren. Der WR betont ausdrücklich, dass die Beurteilung der wissenschaftlichen Qualität alleine Aufgabe der Universität sei. Der Beirat solle nur aus „religiösen Gründen“Einwände gelten machen können. Was unter den möglicherweise konfliktträchtigen „religiösen Gründen“ zu verstehen ist, bleibt jedoch im Dunkeln. ${ }^{7}$

\subsection{Wer vertritt die Muslime in den Beiräten?}

Wenn in den vergangenen zwei Dekaden die Frage nach einem repräsentativen Ansprechpartner auf muslimischer Seite gestellt wurde, entbrannte stets eine Diskussion darüber, ob die in Deutschland tätigen Dachverbände - der Zentralrat der Muslime in Deutschland (ZMD), der Islamrat für die Bundesrepublik Deutschland (IRD), die Türkisch-Islamische Union (DITIB) und der Verband der Islamischen Kulturzentren (VIKZ) - für sich das Recht reklamieren können, für „die Muslime“ in Deutschland zu sprechen. Kritiker verwiesen stets auf die relativ geringen Mitgliederzahlen der Verbandsorganisationen. Unstrittig sei, dass eine deutliche Mehrheit der ca. 4,3 Millionen Muslime Deutschlands in keiner der genannten Organisationen bzw. Unterorganisationen Mitglied sei. Gestützt wird diese Sicht der Dinge auch durch die Ergebnisse der repräsentativen Studie „Muslimisches Leben in Deutschland“, die aufzeigt, dass lediglich 20 Prozent der Muslime in einer religiösen Gemeinde oder einem religiösen Verein organisiert sind. ${ }^{8}$

Eine etwas andere Sicht der Dinge ergibt sich, wenn man von den Moscheegemeinden ausgeht. In Deutschland gibt es ca. 2.600 Gemeinden. Nach eigenen Schätzungen vertreten die im KRM zusammengeschlossenen Dachverbände ca. 85 Prozent der Moscheegemeinden. Gerade die letztgenannten Zahlen zeigen deutlich, dass die Dachverbände, trotz der relativ geringen Mitgliedszahlen, das

\footnotetext{
${ }^{7}$ Vgl. ebd., S. $79 \mathrm{ff}$.

${ }^{8}$ Vgl. BAMF 2009, S. 343. Vgl. zudem den Beitrag von Martina Sauer und Dirk Halm (S. 389-417) in diesem Sammelband.
} 
gemeindliche Alltagsleben in einem erheblichen Ausmaß mitverantworten. Gerade diese unbestrittene Tatsache führte dazu, dass den Dachverbänden in den Beiräten erhebliche Stimmenanteile überlassen werden sollen.

\subsection{Beispiele Hochschule}

\subsubsection{Ordnung des konfessionellen Beirats für Islamische Theologie der Westfälischen Wilhelms-Universität Münster}

Zwischenzeitlich haben nahezu alle universitären Zentren für Islamische Theologie Ordnungen für Beiräte erlassen. Hierbei folgte man in allen wesentlichen Punkten den Empfehlungen des WR. Exemplarisch angeführt sei hier die „Ordnung des konfessionellen Beirats für Islamische Theologie der Westfälischen Wilhelms-Universität Münster", 9 die der Senat der Universität Münster im Dezember 2011 nach kontrovers verlaufender Diskussion verabschiedete. Die elf Paragraphen umfassende Ordnung sichert dem Beirat in drei Punkten erhebliche Mitspracherechte zu. So muss die Universität vor der Einrichtung oder Änderung eines theologischen Studiengangs sowie bei allen „bekenntnisrelevanten Fragen" das Placet des Beirats einholen. Gleiches gilt für den Erlass oder die Änderung von Studien- und Prüfungsordnungen. In beiden Fällen kann das Einverständnis nur „aus religiösen Gründen“ verweigert werden. Was hierunter im Einzelnen zu verstehen ist, wird in den Empfehlungen des WR nicht ausgeführt. Erheblich sind ferner die in der Ordnung eingeräumten Mitwirkungsmöglichkeiten bei Personalentscheidungen. Die Universität muss vor „der Berufung oder Anstellung einer Dozentin/eines Dozenten mit selbstständigen Lehraufgaben“ das Einverständnis des Beirats einholen. Diese Regelung gilt auch bei der Erteilung eines Lehrauftrags. Verweigert werden kann auch hier das Einverständnis nur aus religiösen Gründen, die sich „auf Lehre oder Lebenswandel“ des Betroffenen beziehen. Schließlich verfügt der Beirat über die Möglichkeit, nachträglich die Lehrtätigkeit einer Dozentin/eines Dozenten aus „religiösen Gründen“ zu beanstanden. In einem solchen Fall verpflichtet sich die Universität dazu, dass die betroffene Person nicht mehr im Bereich der Islamischen Theologie unterrichtet. ${ }^{10}$

\footnotetext{
${ }^{9}$ Vgl. Westfälische Wilhelms-Universität Münster 2011.

${ }^{10} \mathrm{Vgl}$. ebenda.
} 
Angesichts dieser beträchtlichen Mitwirkungsmöglichkeiten stellt sich die Frage nach den Entscheidungsträgern bzw. den Mitgliedern des Beirats. Die Ordnung des Konfessionellen Beirats sieht insgesamt acht Mitglieder vor. Vier Mitglieder entsendet der Koordinationsrat der Muslime (KRM), vier weitere Mitglieder umfassen „zwei Persönlichkeiten des öffentlichen Lebens“ und „zwei Religionsgelehrte". Die vier verbandsunabhängigen Mitglieder sind vom KRM und der Universität einvernehmlich zu bestimmen. Sofern eine Einigung nicht erzielt werden kann, benennt die Universität eine Person aus der Liste des KRM und der KRM eine Person aus der Liste der Universität. ${ }^{11}$

Kontrovers diskutiert wurde insbesondere die Rolle des KRM. Angesichts der Tatsache, dass der KRM keine eigene Rechtspersönlichkeit besitzt und damit als ein informeller Zusammenschluss von Dachverbänden angesehen werden muss, in dem die DITIB über ein Vetorecht verfügt, erscheint eine so weitreichende Zusammenarbeit nicht unbedenklich. Hinzu kommt, dass die Regelungen über die Mitgliedschaft den großen, eher konservativ orientierten Verbänden de facto die Mehrheit sichern. Angesichts dieses Sachverhalts stieß die Ordnung universitätsintern auf Widerstand. Nach Angaben der Westfälischen Nachrichten mussten selbst der Senatsvorsitzende, Janberd Oebbecke, gemeinsam mit der Uni-Rektorin, Ursula Nelles, einräumen, dass die Ordnung „keine wirklich gute Lösung " darstelle. $^{12}$

\subsubsection{Konfessioneller Beirat für Islamische Theologie der Universität Tübingen}

Über eine viel diskutierte Beiratskonstruktion verfügt auch die Universität Tübingen. Dort soll ein siebenköpfiger Beirat, ähnlich wie in Münster, bei diversen Institutsangelegenheiten umfassend mitwirken. In Tübingen verzichtete die Universität auf eine direkte Mitwirkung des KRM. Stattdessen kooperiert die Universitätsleitung direkt mit in Baden-Württemberg tätigen islamischen Spitzenverbänden, die insgesamt fünf von sieben Mitgliedern entsenden. Von herausragender Bedeutung ist die DITIB, die gleich drei Beiratsmitglieder benennen durfte. Der VIKZ und die Islamische Gemeinschaft der Bosniaken (IGBD)

\footnotetext{
${ }^{11}$ Vgl. ebenda.

${ }^{12}$ Vgl. Westfälische Nachrichten vom 07.12.2011.
} 
entsenden jeweils ein Beiratsmitglied. Zwei nichtorganisierte Mitglieder werden vom Rektor vorgeschlagen.

Die Zusammensetzung des Beirats konnte nicht einvernehmlich zwischen Universität und muslimischen Organisationen geregelt werden. Die Islamische Gemeinschaft Baden-Württemberg (IGWB), die nach Eigenangaben ca. ein Drittel der Moscheegemeinden des Landes vertritt und an den Vorbereitungsgesprächen teilgenommen hatte, wurde aufgrund einer Intervention des Bundesbildungsministeriums ausgeschlossen. Hintergrund waren nach Angaben des Rektors, Bernd Engler, „verfassungsrechtliche Bedenken“. Stein des Anstoßes ist vor allem die Mitgliedschaft der Islamischen Gemeinschaft Milli Görüs (IGMG) in der IGBW, die seit vielen Jahren vom Verfassungsschutz beobachtet wird. Vor allem dieser Vorgang zeigt, dass die Rechtsstellung der Beiratskonstruktionen als instabil und prekär anzusehen ist. Grundsätzlich gelten sollte, dass staatliche Interventionen in Besetzungsangelegenheiten theologischer Beiräte eine inakzeptable Option darstellen sollten. Hinzu kommt, dass andernorts - so in Münster - die IGMG als Gesprächspartner explizit nicht ausgeschlossen wurde. Diese kann über den KRM direkte Mitwirkungsmöglichkeiten im Beirat wahrnehmen.

Durchaus problematisch ist darüber hinaus die Machtfülle, welche die DITIB in Institutsangelegenheiten ausüben kann. Auch wenn bei der DITIB, insbesondere im hessischen Landesverband, eine begrüßenswerte Tendenz zur Eigenständigkeit erkennbar ist, wird die Agenda der Organisation nach wie vor in einem erheblichen Ausmaß von der türkischen Administration mitbestimmt. Sichtbarer Ausdruck dieses Sachverhalts sind immer noch die Botschaftsräte für Religionsangelegenheiten der Türkischen Republik, die bislang das Amt des Vorstandsvorsitzenden bekleideten. Fortgesetzt wird diese Tradition derzeit von dem Theologen und Botschaftsrat Ali Dere, der in der Türkei als Abteilungsleiter für DIYANET tätig war. Ferner sind die ca. 600 Imame zu erwähnen, die von der türkischen DIYANET nach Deutschland entsandt werden, um in DITIBGemeinden ihre Dienste zu verrichten. Auch diese müssen als hauptamtliche Beschäftigte der türkischen Religionsbehörde Direktiven aus der Türkei befolgen.

In Tübingen erstreckte sich der Einfluss der DITIB nicht nur auf den konfessionellen Beirat, in dem die Organisation gleich drei Sitze für sich beanspruchen konnte, sondern auch auf die Berufungskommission. Nach verlässlichen 
Angaben einer Person, die an den Bewerbungsgesprächen teilgenommen hat, war zumindest in einem Fall der Botschaftsrat Ali Dere beteiligt. Diese Tatsache ist durchaus in zweifacher Hinsicht pikant. Erstens hat im Regelfall eine Religionsgemeinschaft auf die fachliche Auswahl von Kandidatinnen und Kandidaten keinen Einfluss. Zweitens muss kritisch nachgefragt werden, was ein weisungsgebundener Diplomat des türkischen Staates in einer unabhängigen Berufungskommission einer deutschen Universität zu suchen hat. Einer unabhängigen bzw. eigenständigen Islamischen Theologie ist dieser Umstand wenig zuträglich.

\section{Beiräte für den islamischen Religionsunterricht}

Da nach Auffassung der Bildungsministerien der Länder die in Deutschland tätigen islamischen Organisationen nicht den Status einer Religionsgemeinschaft im Sinne des Religionsverfassungsrechts unstrittig erfüllen, bemühen sich aktuell Nordrhein-Westfalen und Niedersachsen um Übergangsmodelle, die die Einführung eines islamischen Religionsunterrichts im Sinne des Artikel 7 Abs. 3 GG ermöglichen sollen. Handlungsleitend waren die Schlussfolgerungen des Zwischenresumees der 3. Plenarsitzung der Deutschen Islamkonferenz vom 13.08.2008, in denen es heißt:

Wegen der besonderen Bedeutung des Religionsunterrichts für die Religionsfreiheit der Schüler und Eltern sollte seine Einführung bei Bedarf nicht daran scheitern, dass die Qualifikation einer Organisation als Religionsgemeinschaft noch nicht endgültig feststeht. In solchen Fällen ist es als Übergangslösung zu einem Religionsunterricht nach Art. 7 Abs. 3 GG denkbar, mit im Land verbreiteten Organisationen zu kooperieren, die Aufgaben wahrnehmen, welche für die religiöse Identität ihrer Mitglieder wesentlich sind. ${ }^{13}$

Die konkrete Umsetzung dieser Empfehlung geschieht in Nordrhein-Westfalen und in Niedersachsen - ähnlich wie an den bereits aufgeführten Universitäten - mit Beiräten.

In Nordrhein-Westfalen wurde für die Einführung des islamischen Religionsunterrichts am 22. Dezember 2011 eigens ein Gesetz erlassen, das unter anderem die Mitwirkungsrechte des Beirats regelt.

\footnotetext{
${ }^{13}$ Deutsche Islam Konferenz (DIK) 2008, Berlin, S. 27.
} 
Der Beirat stellt fest, ob der Religionsunterricht den Grundsätzen im Sinne des Artikels 7 Absatz 3 Satz 2 Grundgesetz entspricht. Er ist an der Erstellung der Unterrichtsvorgaben, der Auswahl der Lehrpläne und Lehrbücher und der Bevollmächtigung von Lehrerinnen und Lehrern zu beteiligen. Eine ablehnende Entscheidung ist nur aus religiösen Gründen zulässig, die dem Ministerium schriftlich darzulegen sind. ${ }^{14}$

Bei der Größe und Zusammensetzung des Beirats orientiert man sich weitgehend am Beispiel der Westfälischen Wilhelms-Universität Münster. Der Beirat für den islamischen Religionsunterricht hat gleichfalls acht Mitglieder. Vier theologisch oder religionspädagogisch qualifizierte Vertreterinnen werden „von den islamischen Organisationen in Nordrhein-Westfalen oder von deren Zusammenschluss bestimmt“. Überdies umfasst der Beirat zwei muslimische Persönlichkeiten des öffentlichen Lebens und zwei muslimische Religionsgelehrte, die vom Ministerium im Einvernehmen mit den organisierten Muslimen benannt werden. $^{15}$

In Niedersachsen wurden für den Beirat keine neuen gesetzlichen Grundlagen erlassen. Der Beirat konstituierte sich auf der Grundlage einer schriftlich niedergelegten Vereinbarung zwischen dem DITIB Landesverband NiedersachsenBremen und der SCHURA Niedersachsen - Landesverband der Muslime in Niedersachsen e. V. Der Beirat „hat die Aufgabe, die erforderliche Mitwirkung beim Religionsunterricht für die durch ihn repräsentierten Religionsgemeinschaften wahrzunehmen und die staatlichen Behörden bei seiner Durchführung zu unterstützen. "16 Wie in Nordrhein-Westfalen überprüft der Beirat die Übereinstimmung des Religionsunterrichts mit den Grundsätzen der Religionsgemeinschaft. Ferner ist er zuständig für die Erteilung und gegebenenfalls den Entzug einer Lehrerlaubnis. Der Beirat setzt sich aus vier stimmberechtigten Mitgliedern zusammen, die zu gleichen Teilen von der DITIB und der SCHURA entsandt werden. Anders als in Nordrhein-Westfalen ist eine Mitwirkung des Staates bei der Zusammensetzung des Beirats explizit nicht vorgesehen.

\footnotetext{
${ }^{14}$ Gesetz- und Verordnungsblatt (GV. NRW.), Ausgabe 2011, Nr. 34 vom 30.12.2011, S. 725-732.

${ }^{15}$ Ebenda.

${ }^{16}$ Vereinbarung über die Bildung eines Beirats für den islamischen Religionsunterricht in Niedersachsen 2011.
} 


\subsection{Die Beiräte in der Praxis - Beispiel Lehrerlaubnis}

Die Haupttätigkeit der neugegründeten Beiräte bestand bislang in der Ausarbeitung von sogenannten „Idschaza“-Ordnungen und der Durchführung der damit verbundenen Vergabeverfahren. Bereits in der Ausarbeitungsphase der „Idscha$\mathrm{za}^{\text {"17 }}$-Ordnungen zeigte sich, dass die Vorstellungen der beteiligten islamischen Spitzenverbände zum Teil deutlich über die üblichen Mitwirkungsbereiche hinausreichten. Zur Erinnerung: Beiräte und Religionsgemeinschaften haben ausschließlich über die religiöse Eignung der Anwärterinnen und Anwärter zu befinden, die fachliche Seite ist alleinige Sache des Staates. Genau dies sah die DITIB in Niedersachsen offenbar zunächst anders. In einem nichtdatierten Papier mit dem Titel „Vorausgesetzte Kompetenzen der islamischen Religionslehrer/innen nach DITIB “18 listet die DITIB 145 Qualifikationsmerkmale, die Lehrkräfte der Primarstufe und der Sekundarstufen I und II erbringen sollen. Dem Papier nach sollen selbst Lehrkräfte in der Grundschule 35 fachliche Voraussetzungen erfüllen. Bereits die nachfolgend zitierten ersten fünf Punkte dokumentieren die Maßlosigkeit des Anforderungsprofils.

1. Kann den Koran nach den Regeln des Tadschwid rezitieren.

2. Kann die letzten Suren (ab 93) sowie alle Suren und Gebete, die zum Verrichten des Gebets notwendig sind und auch Suren Ya-Sin, Mulk und Naba aus dem Koran auswendig rezitieren.

3. Kennt die Bedeutung der auswendig rezitierten Koranverse.

4. Kann bei Bedarf auf Arabischkenntnisse auf A2 Niveau und Klassisch-ArabischKenntnisse auf B2 Niveau zurückgreifen.

5. Kennt die Hauptthemen des Korans. ${ }^{19}$

Wie nicht anders zu erwarten war, hatten in der Diskussion mit den staatlichen Stellen diese Forderungen keinen Bestand. In der aktuellen „Idschaza“Ordnung vom 05. Juni 2012, für die SCHURA und DITIB verantwortlich zeichnen, werden noch sieben Voraussetzungen genannt, die sich im Kern auf die

\footnotetext{
الإجازة Idschāza bezeichnet die Erlaubnis zur Weitergabe der islamischen Lehre.

${ }^{18}$ Das nichtveröffentlichte Papier liegt dem Autor als PDF-Dokument vor.

${ }^{19}$ Vorausgesetzte Kompetenzen der islamischen Religionslehrer/innen nach DITIB, S. 1. Die Zahlen beziehen sich auf die Anspruchniveaus des Gemeinsamen Europäischen Referenzrahmens für Sprachen (GER): Lernen, lehren, beurteilen.
} 
religiöse Eignung der Anwärterin bzw. des Anwärters beziehen. Die Ordnung verlangt von den Bewerberinnen und Bewerbern

- das „Bekenntnis zum Islam“

- „die Versicherung und Bereitschaft, den Religionsunterricht in Übereinstimmung mit den Lehren des Islam glaubwürdig zu erteilen und in der persönlichen Lebensweise die Grundsätze islamischer Lebensführung ... zu beachten"

- die „aktive Teilnahme am religiösen Leben der islamischen Gemeinschaft“

- und die erfolgreiche Teilnahme an einem „Motivationsgespräch“ mit dem Beirat. ${ }^{20}$

Die Idschaza-Ordnung, die sich weitgehend an kirchlichen Regelungen orientiert, die insbesondere bei der Erteilung der katholischen missio canonica Geltung beanspruchen können, stießen innermuslimisch teilweise auf massive Kritik. Die Erlanger Religionspädagogen Harry Harun Behr, Amin Rochdi und die Religionspädagogin Fahima Ulfat weisen in ihrer umfassenden und theologisch fundierten Kritik zunächst darauf hin, dass das in der Lehrbefugnis vorzufindende Religionsverständnis auf eine kirchenähnliche Stabilisierung des Islam hinauslaufe, die erhebliche Risiken mit sich brächte. Die Verfasser der Ordnung drohen „eben genau das zu gefährden, was sie schützen wollen, nämlich die Authentizität der Lehre und die Integrität ihrer Repräsentanten“. ${ }^{21}$

Massive Kritik äußern die Erlanger Theologen ferner zu den „Idschaza“-Regelungen, die die Lebensführung und das gemeindliche Engagement der Bewerberinnen und Bewerber betreffen. Hierzu heißt es in der „Idschaza“-Ordnung:

c. Versicherung und Bereitschaft, den Religionsunterricht in Übereinstimmung mit den Lehren des Islam glaubwürdig zu erteilen und in der persönlichen Lebensweise die Grundsätze islamischer Lebensführung, die ethisch-moralische Werte umschließen, zu beachten.

d. Aktive Teilnahme am religiösen Leben der islamischen Gemeinschaft.

e. Die Teilnahme wird nachgewiesen durch ein Empfehlungsschreiben - als Vordruck - des gewählten Vorstandes und des Imams einer Moschee, sowie einer eigenen Darstellung des Bewerbers oder der Bewerberin über die Art der Beteiligung

\footnotetext{
${ }^{20}$ Idschaza-Ordnung Niedersachsen 2012.

${ }^{21}$ Harry Harun Behr, Amin Rochdi und Fahima Ulfat 2012, S. 19.
} 
am Gemeindeleben. Der Beirat kann ggf. die Beibringung ergänzender Unterlagen verlangen. $^{22}$

Diese Regelungen und deren „investigativer Charakter“ halten die Erlanger Religionspädagogen für problematisch. Der Text lasse ein grundsätzliches Misstrauensvotum erkennen. Dabei sollte es eher umgekehrt sein. Eine Lehrkraft sollte hoffen dürfen, im Beirat einen solidarischen Partner zu finden. Stattdessen „tritt ihr neben einer gegen den Islam konditionierten Öffentlichkeit, einer sensibilisierten Dienstaufsicht, einem kritisch eingestelltem Kollegium (...) nun noch ein weiterer Golem gegenüber“. ${ }^{23}$ Darüber hinaus widerspräche die Abfrage zur vorbildlichen Lebensführung den hier üblichen freiheitlichen Standards. Mit dem Verweis auf Muhammads glaubenseifrigen Gefährten Umar ibn al-Chattab, dem der Prophet untersagte, über die Mauer zu spähen, um die Lebensstile der Gemeindemitglieder zu überprüfen, kommen die Erlanger schließlich zu dem Urteil, dass kein Fall bekannt sei, in dem Muhammad Gemeindemitglieder derart auf den Prüfstand gestellt habe, wie es die „Idschaza“-Ordnung vorsehe. Insgesamt betrachtet erhebe der Text einen „maturidischen Standard“, der sich durch einseitige Engführung der Quelltexte auszeichne und mit der ursprünglichen Weite islamischer Theologie und Philosophie wenig gemein habe. Dies ist nach Auffassung der Erlanger sehr bedauerlich, denn die islamische Theologie in Deutschland gewinne ihre Attraktivität durch die Pluralität eines islamischen Religionsverständnisses, dass von dem „staubigen Kerkern“ der „Religionsbürokratie“ befreit sei. Mit der Lehrbefugnisordnung marschiere man „ohne Not in die Gegenrichtung los“. ${ }^{24}$

Auch in Nordrhein-Westfalen ist der Beirat für die Erteilung der "Idschaza“ bzw. Bevollmächtigung zuständig. Die ersten Vergabeverfahren zur Bevollmächtigung verliefen nach Lage der Dinge intransparent und nicht ganz reibungslos. Als ungewöhnlich ist zunächst der Umstand anzusehen, dass nahezu vierzig Vergabeverfahren auf der Grundlage einer nicht veröffentlichten Verfahrensordnung durchgeführt wurden. Selbst interessierten Personen - so dem FAZJournalisten Hermann Horstkotte - wurde bis August 2012 die Einsicht ver-

\footnotetext{
${ }^{22}$ Idschaza-Ordnung, S. 2.

${ }^{23}$ Harry Harun Behr, Amin Rochdi und Fahima Ulfat 2012, S. 20.

${ }^{24}$ Ebenda, S. 21
} 
wehrt. ${ }^{25}$ Die Publikation der Verordnung erfolgte erst am 03.09.2012. Warum Beirat und Ministerium an diesem Punkt Geheimniskrämerei betrieben, ist nicht ganz nachvollziehbar.

Durchaus pikante Details wurden ferner aus den jeweils halbstündigen Einzelgesprächen berichtet, welches die künftigen Religionspädagoginnen und Pädagogen durchlaufen mussten. Nach Recherchen von Hermann Horstkotte ging es hierbei auch um die Eindeutschung der Fachtermini, die Hadsch und weitere fachliche Fragen. ${ }^{26}$ Eine weitere seriöse Quelle berichtet darüber hinaus, dass Kandidatinnen und Kandidaten ihre Korankompetenz durch das zügige Auffinden von Suren unter Beweis stellen mussten. Diese Vorgehensweise irritierte nicht nur die Lehrkräfte; sie entbehrt sogar jeglicher gesetzlicher Grundlage. Auf der Grundlage des modifizierten Schulgesetzes darf der Beirat ausschließlich über die religiöse Eignung befinden. Die Überprüfung der fachlichen Kompetenzen ist alleine Sache des Staates.

Für weitere Irritationen sorgte im August 2012 ein Brief des Beirats, der die bereits zugelassenen Lehrkräfte mit Fristsetzung aufforderte, dem Beirat bestehende Mitgliedschaften in Moscheegemeinden mitzuteilen. Auch in diesem Fall muss konstatiert werden, dass der Beirat, der sich scheinbar wesentlich als religiöse Kontrollinstanz begreift, seine Befugnisse in einem nicht hinnehmbaren Ausmaß überschreitet.

\section{Fazit}

Die aufgeführten Beispiele zeigen, dass die Implementierung von Beiräten an Universität und Schule zahlreiche Problemstellungen mit sich bringt. Erste Unklarheiten bestehen bereits bei der Zusammensetzung der Beiräte. Das Tübinger Beispiel demonstriert, dass der Staat unliebsame Organisationen ausschließen kann. Für einen unabhängigen theologischen Beirat ist ein solcher Akt staatlicher Einmischung kaum hinnehmbar. Fragwürdig ist ferner die herausragende Position der islamischen Spitzenverbände, insbesondere der DITIB, die in zahlreichen inhaltlichen und personellen Fragen erhebliche Mitwirkungsrechte geltend machen können. $\mathrm{Zu}$ fragen ist in diesem Zusammenhang auch nach den

\footnotetext{
${ }^{25}$ Vgl. Hermann Horstkotte 2012.

${ }^{26}$ Vgl. ebenda.
} 
Mitwirkungsmöglichkeiten kleinerer muslimischer Organisationen und nichtorganisierter Muslime. Die bislang getroffenen Regelungen sind nicht wirklich überzeugend, da alle Beiräte die faktische Pluralität islamischen Lebens nur unzureichend zur Abbildung bringen. Unklarheiten bestehen schließlich auch bei den Kompetenzen der Beiräte. Die angeführten Beispiele zu den jüngsten Beiratsaktivitäten im Kontext der Vergabe von Bevollmächtigungen zeigen deutlich, dass einige Verbandsvertreter umfassende Kontrollbefugnisse für sich reklamieren.

\section{Literatur}

BAMF - Bundesamt für Migration und Flüchtlinge (Hrsg.), Muslimisches Leben in Deutschland. Im Auftrag der Deutschen Islamkonferenz, Nürnberg 2009.

Behr, Harry, Harun; Rochdi, Amin und Ulfat, Fahima, Zur Diskussion um die Lehrbefugnisornung, in: Zeitschrift für die Religionslehre des Islam, Heft 11, Mai 2012.

Deutsche Islam Konferenz (DIK), Zwischen-Resümee der Arbeitsgruppen und des Gesprächskreises, Vorlage für die 3. Plenarsitzung der DIK, 13. März 2008, Berlin 2008.

Horstkotte, Hermann, Auf dem Schulweg zum Staatsislam, FAZ vom 24.07.2012.

Kiefer, Michael, „Islamische Studien“ an deutschen Universitäten - Zielsetzungen, offene Fragen und Perspektiven, in: Aus Politik und Zeitgeschichte, Nr. 13/14, 2011.

WR - Wissenschaftsrat, Empfehlungen zur Weiterentwicklung von Theologien und religionsbezogenen Wissenschaften an deutschen Hochschulen, Berlin 2010.

\section{Internetquellen}

Brunner, Rainer: Die Empfehlungen des Wissenschaftsrats zur Einführung des Fachs „Islamische Studien“ aus der Sicht eines Islamwissenschaftlers, unter: http://www. wissenschaftsrat.de/download/archiv/Brunner.pdf (04.08.2012).

Idschaza-Ordnung des Beirat für den islamischen Religionsunterricht in Niedersachsen, unter: http://beirat-iru-n.de/app/download/5780358776/ljaza-Ordnung + Niedersachsen + Fssg + 27.01.12.pdf (18.08.2012).

Westfälische Nachrichten vom 07.12.2011, unter: http://www.wn.de/Muenster/2011/ 12/Hitzige-Debatte-um-Islambeirat-an-Uni-Senat-Zweifel-an-Zusammensetzung (13.08.2012).

Westfälischen Wilhelms-Universität Münster, Ordnung des konfessionellen Beirats für Islamische Theologie vom 21. Dezember 2011, unter: http://www.uni-muenster.de/ imperia/md/content/wwu/ab_uni/ab2012/ausgabe03/beitrag_02.pdf (13.08.2012). 
Vereinbarung über die Bildung eines Beirats für den islamischen Religionsunterricht in Niedersachsen, unter: http://beirat-iru-n.de/app/download/5778852067/Beiratsvereinba rung + 17.01.11.pdf (17.08.2012). 